\title{
Improvement of Evaluation of Replacement Cost of a Fusion Power Plant ${ }^{*}$
}

\author{
Takuya GOTO, Hiroyasu UTOH ${ }^{1)}$, Youji SOMEYA ${ }^{1)}$, Makoto NAKAMURA ${ }^{1)}$, \\ Ryoji HIWATARI ${ }^{2)}$ and Kenji TOBITA ${ }^{1)}$ \\ National Institute for Fusion Science, 322-6 Oroshi-cho, Toki 509-5292, Japan \\ 1) Japan Atomic Energy Agency, 2-168 Oaza-Obuchi-Aza-Omotedate, Rokkasho 039-3212, Japan \\ ${ }^{2)}$ Central Research Institute of Electric Power Industry, 2-11-1 Iwado-kita, Komae 201-8511, Japan
}

(Received 10 December 2013 / Accepted 24 July 2014)

\begin{abstract}
A new model to evaluate the plant availability of a fusion reactor by considering the required cooling time before starting the scheduled replacement of in-vessel components was developed and incorporated in the tokamak system design code TPC-SCONE. Plant availability strongly depends on the electric output and decreases with increasing electric output. The plant availability does not much improve by advances in the physics or engineering design conditions. Consequently, the effect of the increase in the electric output on reducing the cost of electricity is diminished. Thus, proper selection of the electric output of a fusion reactor (especially for a DEMO reactor) is likely required to achieve the plant availability target.
\end{abstract}

(C) 2014 The Japan Society of Plasma Science and Nuclear Fusion Research

Keywords: reactor design, system design code, cost model, plant availability, time required for replacement

DOI: $10.1585 /$ pfr.9.3405140

\section{Background}

The research and development of fusion energy is at the conceptual design stage of a DEMO reactor, which is anticipated to improve the financial viability of a fusion power plant. This means that economy is a key factor in the design parameters of a DEMO reactor. For estimating the cost of a fusion reactor, the Generomak model [1] and its revisions have been widely used. In the Generomak model, capital cost of a fusion island is directly calculated by mass-cost analysis based on the amount and mass unit cost of the materials of each component. On the other hand, estimation of other costs is based on the design of a pressurized water reactor (PWR). Thus, it does not fully reflect fusion-specific conditions and has room for improvement. One of the big differences between a PWR and a fusion power plant is the scheduled replacement of the invessel components. In the Generomak model, the cost of the scheduled replacement is reflected as the capital cost of the replacement components. On the other hand, plant availability is simply the input, whereas the time required for the replacement is not directly considered. However, the time required for the replacement may significantly affect plant availability. In particular, the required cooling time after the shutdown of the plasma operation and before starting the replacement is important. Because the primary coolant system of the replaced components should be stopped during the replacement, the temperature of the components increases until the heat removal capacity by

author's e-mail: goto.takuya@LHD.nifs.ac.jp

*) This article is based on the presentation at the 23rd International Toki Conference (ITC23). passive or other indirect cooling methods balance the heat production by the decay heat. Thus, adequate cooling time is required to suppress the maximum temperature below the allowable level. This residual decay heat strongly depends on the primary design parameters in systems codes (e.g., reactor size, fusion power, blanket thickness) and can be quantitatively evaluated. Therefore, a new model to evaluate the required cooling time was developed and parametric scans were carried out.

\section{Calculation Model}

In this study, the tokamak systems code TPC [2,3] coupled with the TF coil design code SCONE [4] was used (TPC-SCONE). For cost evaluation, the cost model of the tokamak systems code FUSAC [5] was partly modified and incorporated in the TPC-SCONE code. The required cooling time of the in-vessel components before starting the replacement is estimated in the following manners. In the design study of the tokamak reactor SlimCS with fusion power of $\sim 3 \mathrm{GW}[6]$, the maximum temperature at the surface of the high-temperature shield behind the blanket after stopping the primary coolant system of the in-vessel components was evaluated as a function of the cooling time after the shutdown of the plasma operation [7] (see Fig. 1). Forced air cooling of the replaced component is assumed after the stopping of primary coolant system. The maximum temperature depends on the total decay heat of the adjacent blanket. The total decay heat is calculated by integrating the decay heat density profile over the entire volume of the blanket. The decay heat density is a function of 


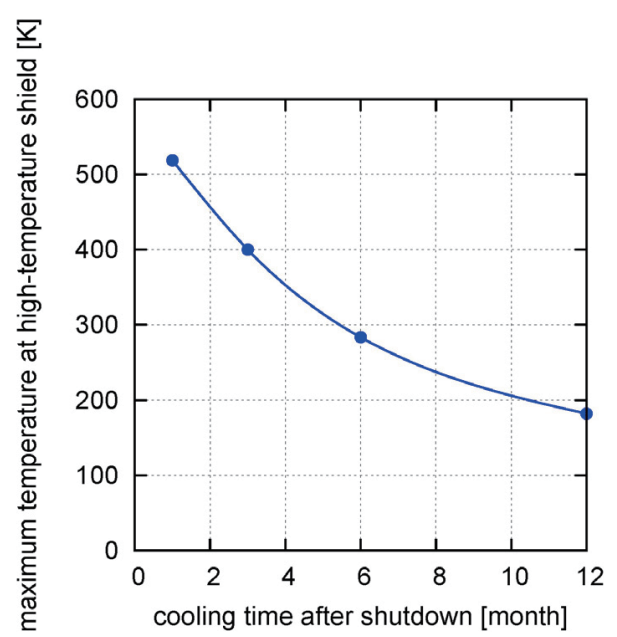

Fig. 1 Maximum temperature of the high-temperature shield of the SlimCS reactor after the stopping of the primary coolant system as a function of the cooling time after the shutdown of the plasma operation in the case of fusion power of $3 \mathrm{GW}$.

the neutron flux on the blanket and the thickness and material composition of the blanket. Here we only consider blankets with the same thickness and material composition as those of SlimCS. In this case, the total decay heat of the blanket can be scaled from the analysis of SlimCS by multiplying the ratio of the blanket volume and the ratio of the neutron flux (neutron wall load) on the blanket. The use of reduced activation ferritic steel F82H for the high-temperature shield is considered; hence, the maximum temperature needs to be suppressed below $550^{\circ} \mathrm{C}$ in order to reuse. We define the required cooling time as that needed for the maximum temperature to reach $550^{\circ} \mathrm{C}$.

\section{Calculation Result}

Using the TPC-SCONE code with the developed model, parametric scans were conducted. Five parameters were considered: the reactor major radius $R_{p}$, the safety factor $q_{95}$, the normalized beta $\beta_{N}$, the averaged temperature $\langle T\rangle$, and the TF coil thickness $d_{\mathrm{TF}}$. The range of these parameters is summarized in Table 1. Other input parameters are fixed with respect to those of SlimCS, as shown in Table 2. For the TF coil design, the use of $\mathrm{Nb}_{3} \mathrm{Sn}$ superconductor and ITER-relevant engineering design constraints were assumed. We also assumed the required time for the replacement to be three months (two months for the replacement work and one month for the testing of new components). Plant availability was calculated as the ratio of the time of the plasma operation to the total time of one operation cycle (the sum of plasma operation time, cooling time, and the time for the replacement).

Figure 2 shows the dependence of the COE (normalized by that of the calculation result with input parameters equivalent to those of SlimCS for which the net electric output is $0.87 \mathrm{GW}$ ) and the plant availability on the
Table 1 Range of input parameters.

\begin{tabular}{|l|l|}
\hline \multicolumn{1}{|c|}{ parameter } & \multicolumn{1}{c|}{ range } \\
\hline major radius $R_{p}[\mathrm{~m}]$ & $5-10$ \\
\hline safety factor $q_{95}$ & $3.0-5.4$ \\
\hline normalized beta $\beta_{N}$ & $2.0-4.4$ \\
\hline averaged temperature $\langle T\rangle[\mathrm{keV}]$ & $17-23$ \\
\hline TF coil thickness $d_{\mathrm{TF}}[\mathrm{m}]$ & $1.2-2.4$ \\
\hline
\end{tabular}

Table 2 Fixed input parameters.

\begin{tabular}{|l|l|}
\hline \multicolumn{1}{|c|}{ parameter } & \multicolumn{1}{|c|}{ value } \\
\hline elongation $\kappa$ & 2.0 \\
\hline triangularity $\delta$ & 0.35 \\
\hline plasma aspect ratio $A$ & 2.6 \\
\hline density profile factor $\alpha_{n}$ & 0.5 \\
\hline temperature profile factor $\alpha_{T}$ & 0.75 \\
\hline alpha particle fraction $f_{\alpha}$ & 0.07 \\
\hline impurity (argon) fraction $f_{\text {Ar }}$ & 0.0012 \\
\hline inboard/outboard blanket thickness [m] & $0.3 / 0.5$ \\
\hline shield thickness [m] & 0.5 \\
\hline blanket coverage & 0.7 \\
\hline neutron multiplication factor & 1.4 \\
\hline thermal efficiency $\eta_{\text {th }}$ & 0.35 \\
\hline fixed charge rate (FCR) & 0.12 \\
\hline
\end{tabular}

net electric output in the case of the $10 \mathrm{MWa} / \mathrm{m}^{2}$ (150 dpa) neutron fluence limit of the in-vessel components, which is considered as the limit for $\mathrm{F} 82 \mathrm{H}$. The design points that have space for center solenoid coils larger than $0.7 \mathrm{~m}$ are plotted, considering the pulsed operation with inductive current drive as an option for a DEMO reactor. We see that plant availability monotonically decreases with increasing net electric power. Because these design points include various reactor sizes and magnetic field strengths, it was found that the COE and plant availability are not sensitive to the engineering design conditions. Furthermore, improvements in the core plasma performance (e.g., Greenwald density limit ratio $f_{\mathrm{GW}}$, confinement improvement factor $H_{H}$, and normalized beta $\beta_{N}$ ) have limited effect. Because of the residual decay heat, the dominant factor for determining the time required for the replacement depends not on the core plasma performance or the neutron flux on the blanket but on the total neutron yield. In this case, the degradation of plant availability approximately $10 \%$ of the net electric output of $P_{\mathrm{e} \text {,net }}=1 \mathrm{GWe}$, which is the anticipated target for a commercial power plant. Although the reduction in the COE with increasing the electric output (merit of scale) is diminished, larger electric output is better from the viewpoint of the COE. However, the mechanism that determines the divertor module lifetime is not fully understood and is probably shorter than that of the blanket, especially at the early stage of DEMO operation. Therefore, we performed similar parameter scans for fluence limits of the in-vessel components of $5 \mathrm{MWa} / \mathrm{m}^{2}$ (75 dpa for F82H, Fig. 3) and $2 \mathrm{MWa} / \mathrm{m}^{2}$ (30 dpa for F82H, 

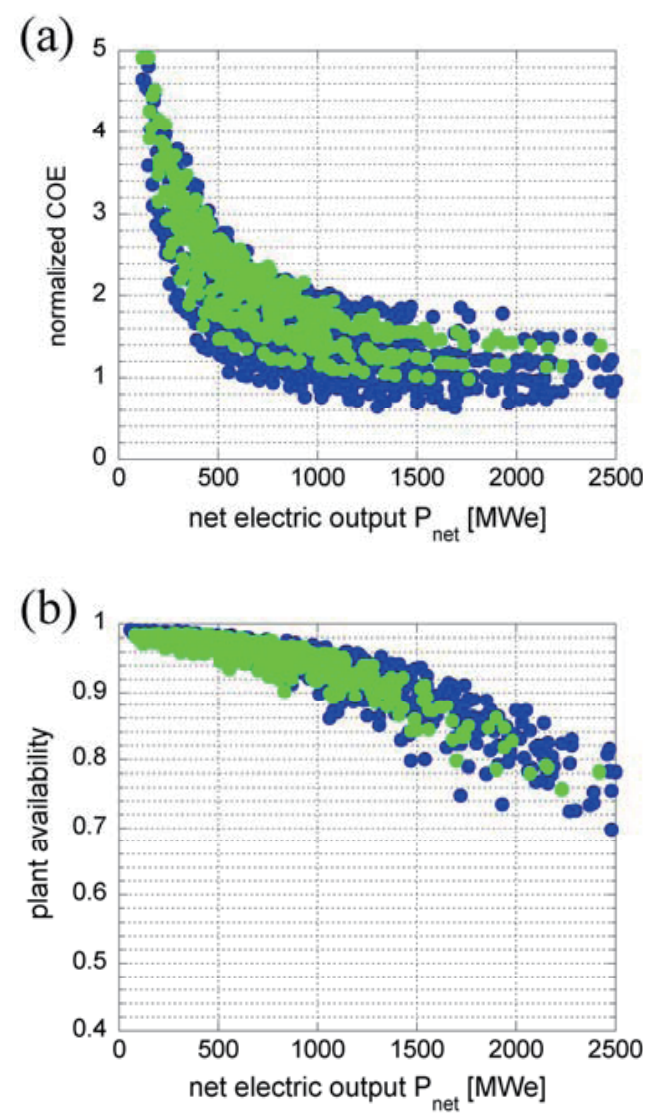

Fig. 2 Dependence of (a) COE normalized by that of the calculation result with input parameters equivalent to those of SlimCS and (b) plant availability on the net electric output in the case of neutron fluence limit of blankets of $10 \mathrm{MWa} / \mathrm{m}^{2}$ (150 dpa for $\mathrm{F} 82 \mathrm{H}$ ) for design points that satisfy $R_{\mathrm{CS}} \geq 0.7 \mathrm{~m}, f_{\mathrm{GW}} \leq 1.2$, and $H_{H} \leq 1.5$. Green points correspond to the design with $R_{\mathrm{CS}} \geq 0.7 \mathrm{~m}, f_{\mathrm{GW}} \leq 1.0$, $H_{H} \leq 1.2$, and $\beta_{N} \leq 3.0$.

Fig. 4). Even for fluence limit of $2 \mathrm{MWa} / \mathrm{m}^{2}$, the COE decreases with increasing electric output for $P_{\mathrm{e} \text {,net }} \leq 1 \mathrm{GWe}$. However, plant availability is below $80 \%$ for conservative physics conditions, and the absolute value of COE is roughly two times larger than that in the case of fluence limit of $10 \mathrm{MWa} / \mathrm{m}^{2}$. Figure 5 shows the dependence of the total plant construction cost on the net electric output in the case of neutron fluence limit of $10 \mathrm{MWa} / \mathrm{m}^{2}$. Because the plant construction cost does not depend on plant availability, the dependence is the same as that in the case of different fluence limits. Although the total plant construction cost has a relatively wide range at the same electric output, the overall trend shows increasing construction cost with increasing net electric output. This trend is clearer for small electric output $\left(P_{\mathrm{e}, \text { net }}<500 \mathrm{MWe}\right)$.

The calculation results suggest that not only the neutron fluence limit of $\sim 10 \mathrm{MWa} / \mathrm{m}^{2}$ of the blanket system but also the lifetime of the divertor module matching the duration determined by the fluence limit are required to achieve plant availability of $\sim 90 \%$ with $1 \mathrm{GWe}$-class net
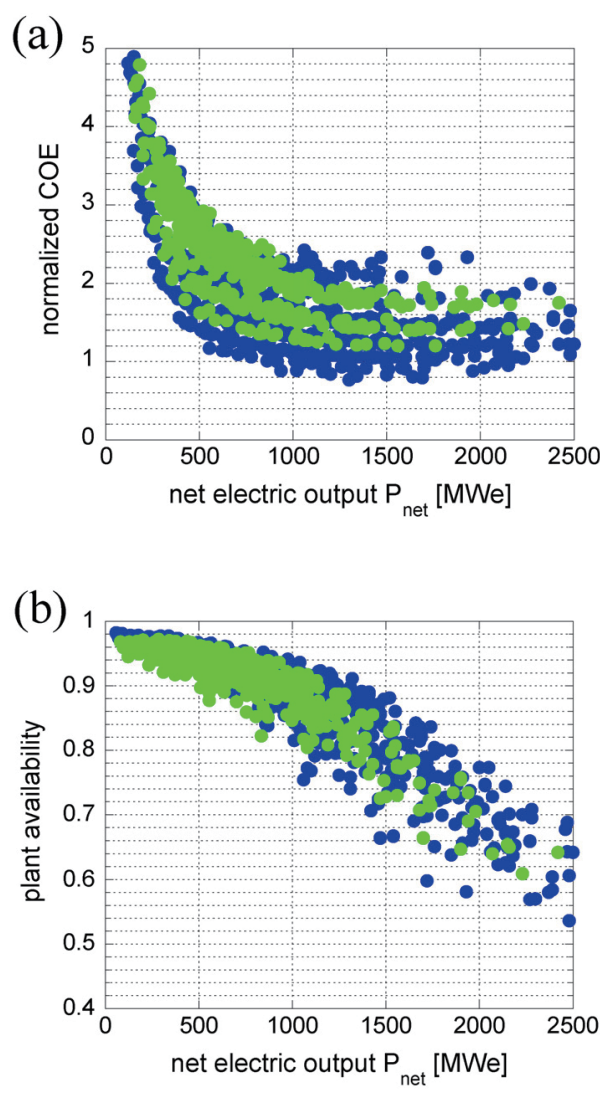

Fig. 3 Dependence of (a) COE normalized by that of the calculation result with input parameters equivalent to those of SlimCS and (b) plant availability on the net electric output for blanket neutron fluence limit of blanket is $5 \mathrm{MWa} / \mathrm{m}^{2}$ ( $75 \mathrm{dpa}$ for $\mathrm{F} 82 \mathrm{H}$ ). Constraints are the same as those in Fig. 2.

electric output. Therefore, increasing the lifetime of the in-vessel components is required to improve the commercial prospects of fusion power plants. Plant availability depends on the frequency of the replacement and the time required for the replacement; thus, the time required for the replacement work should be minimized by optimizing the replacement procedure. On the other hand, decreasing the net electric output is an option for a DEMO reactor because it minimizes the construction cost and improves plant availability. Consequently, the design parameters of a DEMO reactor should be carefully selected considering the mission and operation period of the DEMO reactor.

\section{Conclusion}

To quantitatively evaluate the cost of the scheduled replacement of a fusion reactor, a new calculation model to estimate the time required for the cooling of the invessel components before starting the replacement was developed and parametric scans were carried out using the systems code TPC-SCONE. It was found that plant availability strongly depends on the fusion output and does not much improve by improving the core plasma performance 

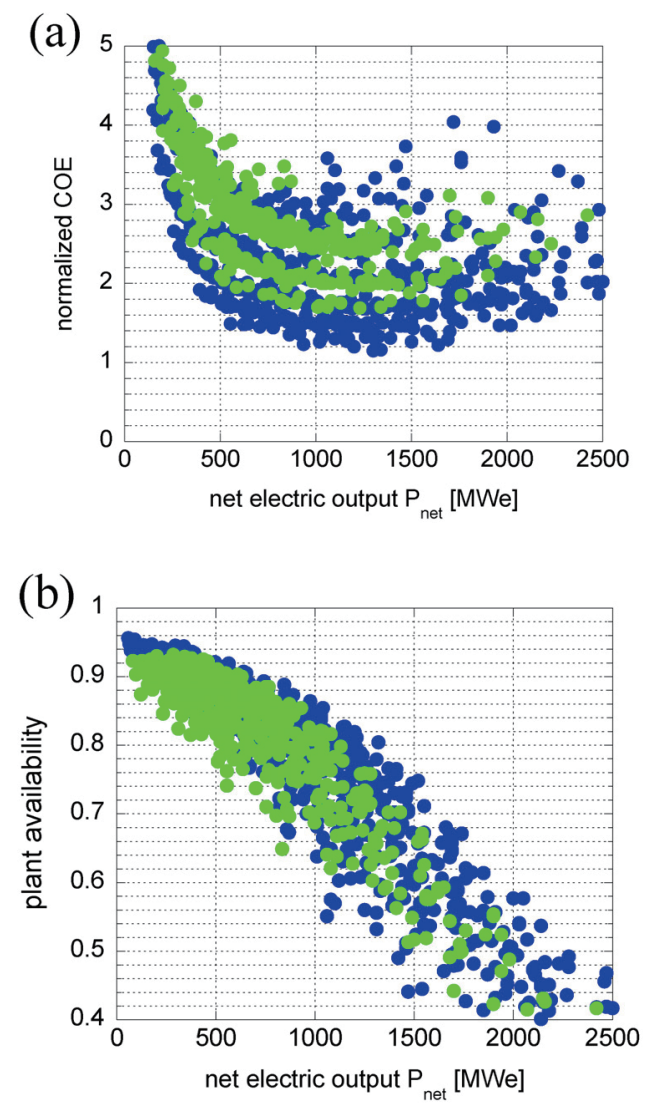

Fig. 4 Dependence of (a) COE normalized by that of the calculation result with input parameters equivalent to those of SlimCS and (b) plant availability on the net electric output for blanket neutron fluence limit of blankets is $2 \mathrm{MWa} / \mathrm{m}^{2}$ (30 dpa for $\mathrm{F} 82 \mathrm{H}$ ). Constraints are the same as those in Fig. 2.

and engineering design constraints. Therefore, improving the lifetime of in-vessel components is indispensable to realize a $1 \mathrm{GWe}$-class commercial fusion power plant. On the other hand, decreasing the net electric output without departing from the mission is an option for a DEMO reactor

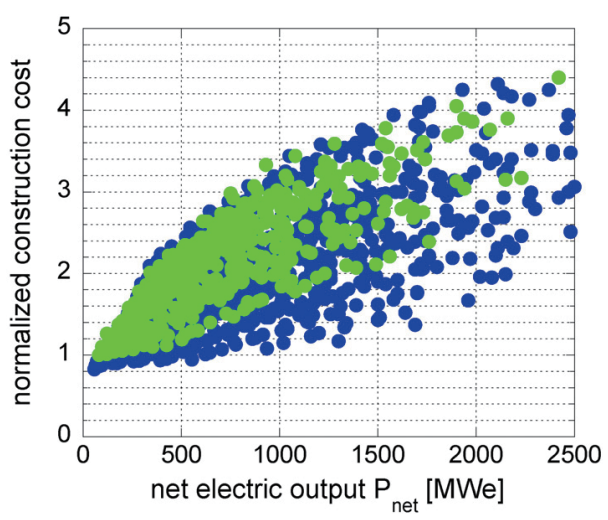

Fig. 5 Dependence of the total plant construction cost (normalized by that of the calculation result with input parameters equivalent to those of SlimCS) on the net electric output. Constraints are the same as those in Fig. 2.

for minimizing the total construction cost and improving plant availability. For more reliable evaluation, the refinement of the cost model (accurate estimation of unit cost of components, detailed modeling of the cost, electric power consumption of fusion-specific system) and design concept (pulsed operation mode, different type of blanket system, the use of advanced materials), and parametric scans over wider range are needed.

\section{Acknowledgement}

This work is supported by the BA DEMO design and safety collaborative research.

[1] J. Sheffield et al., ORNL/TM-9311 (1986).

[2] H. Fujieda et al., JAERI-M 92-178 (1992) (in Japanese).

[3] M. Nakamura et al., Fusion Eng. Des. 87, 864 (2012).

[4] H. Utoh et al., J. Plasma Fusion Res. SERIES 9, 304 (2010).

[5] R. Hiwatari et al., Nucl. Fusion 44, 106 (2004).

[6] K. Tobita et al., Nucl. Fusion 47, 892 (2007).

[7] H. Utoh et al., Nucl. Fusion 53, 123005 (2013). 